\title{
Autologous Mesenchimal Stem Cell Therapy Infertile Patients: A Case Report
}

ISSN: 2640-9666

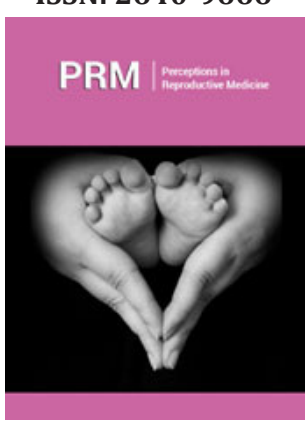

*Corresponding author: Víctor Manuel VH, Women's Health Clinic, Mexico

Submission: 留June 18, 2020

Published: 觜July 21, 2020

Volume 4 - Issue 1

How to cite this article: Jesús Estuardo LI, José Juan GV, Víctor Manuel VH, et al. Autologous Mesenchimal Stem Cell Therapy Infertile Patients: A Case Report. Perception in Reproductive Medicine. 4(1). PRM. 000576. 2020.

DOI: 10.31031/PRM.2020.04.000576

Copyright@ Víctor Manuel VH, This article is distributed under the terms of the Creative Commons Attribution 4.0 International License, which permits unrestricted use and redistribution provided that the original author and source are credited.

\author{
Jesús Estuardo LI, José Juan GV, Alejandro KB, Felipe de Jesús AP, Daniela AR, \\ Carlos DM and Víctor Manuel VH* \\ *Department of Women's Health Clinic, Mexico
}

\begin{abstract}
Background: In Mexico there are 17\% of women of reproductive age with infertility problems, equivalent to 1.4 million couples who need assisted reproductive techniques; 9 to $24 \%$ of these patients present low ovarian response, mainly in women older than 38 years, due to ovarian aging.

Objective: To design a human method to administer autologous adipose tissue mesenchymal stem cells directly into the ovaries to elucidate the underlying mechanisms of ovarian rejuvenation and point the way for the development of future therapies.

Method and Result: We present a case of a 39-year-old woman, classified as low or suboptimal ovarian response. included in group 2, subgroup 2a, of the Poseidon criteria; to which a human method was designed to administer autologous adipose tissue mesenchymal stem cells (AD-MSCs) directly into the ovaries prior to ovarian stimulation; under written informed consent to the patient; It was carried out in a procedure where 14 oocytes were obtained, which were fertilized with donor semen (normozoospermic) and a biopsy was performed of three blastocysts obtained, to carry out a preimplantation genetic study that reported an euploid embryo; This is placed in the mother's uterus in order to achieve pregnancy and a healthy newborn. Once transferred to the mother, the euploid blastocyst, the human gonadotropin hormone beta subunit test was positive $6572 \mathrm{mUI} / \mathrm{mL}$ and it was completed, obtaining a male newborn obtained by caesarean section at 39.2 weeks of healthy gestation with adequate evolution.
\end{abstract}

Conclusion: Stem cell therapy obtained from bone marrow is an alternative for women with low ovarian response to achieve pregnancy, by improving the quality of oocytes; it is the first report in Mexico, in women with low idiopathic ovarian response.

Keywords: Low ovarian response; Infertility; Ovarian aging; Adipose tissue mesenchymal stem cells

\section{Background}

In Mexico there are $17 \%$ of women of reproductive age with infertility problems, which is equivalent to 1.4 million couples need assisted reproduction techniques; from 9 to $24 \%$ of these patients present a low ovarian response, which is defined as the poor obtaining of mature eggs after ovarian stimulation of follicular puncture during in vitro fertilization, which occurs with a higher incidence in women older than 38 years, mainly due to ovarian aging and decreased ovarian reserve. In particular, low responders are not a homogeneous population of patients and their prognosis varies greatly depending on parameters such as age and number of oocytes retrieved [1-5]. Scientific and technological advances in the management and treatment of patients with a low ovarian response continue to be controversial and the criteria for diagnosing it have changed and since 2011 the Bologna criteria and later those of Poseidon [1-5] have been implemented. The Poseidon criteria are divided into 4 categories, based on quantitative parameters, quality, age, number of antral follicles or antimullerian hormone levels, and ovarian response (after standard reference stimulation). Within these criteria, specifically group 1 (those $<35$ years of age) and 2 (those $\geq 35$ years of age), refer to normo-gonadotropic patients, who present antral follicular count (AFC) $\geq 5$ and hormone antimulleriana $\geq 1.2 \mathrm{ng} / \mathrm{mL}$, with subgroups 1 a or 2 a respectively, where $<4$ oocytes are obtained in metaphase II: expanded and lutinized cluster cells, in follicular aspiration and 
subgroups $1 \mathrm{~b}$ and $2 \mathrm{~b}$, respectively where [4-9] oocytes are obtained in AF. These patients are classified as low or suboptimal ovarian response $[2,4,5]$.

The importance of the classification of the low ovarian response is to obtain a timely diagnosis, because it allows strategies that will have better results, and the proposals for patients with low idiopathic ovarian response, are due to high doses of analogs of the hormone releasing hormones. Gonadotropins and cycles longer than 10 days, due to possible follicle-stimulating hormone receptor polymorphisms, less bioactive luteinizing hormone variants or damage to these molecules by free radicals and or environmental contaminants, in addition to lifestyle and nutrition, which could be responsible for low sensitivity to follicle-stimulating hormone; the application of high doses of luteinizing hormone is proposed, which improves inadequate levels of endogenous androgens; and they increase the expression of follicle-stimulating hormone receptors in granulosa cells, which leads to increased ovarian response capacity to follicle-stimulating hormone [6-8]. Mesenchymal stem cell therapy has been considered as a new option to treat female infertility since it allows the use of own eggs to restore ovarian function, which has opened new doors for women with low ovarian response [9-15].

MSCs are cells capable of self-renewal, with a high proliferation rate and the ability to differentiate between different cells of the same organ or embryonic layer (Multipotent) or a single cell type (unipotent). They are obtained from bone marrow, peripheral blood, umbilical cord, adipose tissue, etc., either autologously or from a donor, and have been used in cell therapy for diseases such as Alzheimer's, amyotrophic lateral sclerosis, Huntington's, Parkinson's, cerebral infarctions. and myocardium, spinal cord damage, immune disorders, osteoarthritis, restoration of ovarian function, etc. $[5,16,17,18-20]$. Secrete a wide selection of cytosines, chemokines and growth factors, these characteristics give them anti-apoptotic, anti-inflammatory, pro-angiogenic and immunomodulatory properties, in addition, they help in tissue repair, replacing damaged cells, which makes them highly attractive to the clinic $[12,16,18,21-24]$. Based on this information, a human method was designed to administer autologous adipose tissue mesenchymal stem cells (AD-MSCs) directly into the ovaries. The results may begin to elucidate the underlying mechanisms of ovarian rejuvenation and point the way for the development of future therapies.

\section{Clinical Case}

We present a 39 -year-old woman, weighing $60.5 \mathrm{~kg}$, height $1.66 \mathrm{~m}$, with secondary infertility, 2 episodes, 2 abortions, and antimulleriana hormone levels of $4 \mathrm{ng} / \mathrm{mL}$. He does not have a partner and due to his age vitrification of oocytes is decided. In its first cycle in the clinic, antral follicles were counted on day 2 of the cycle by vaginal ultrasound (Philips, Affiniti $50,7 \mathrm{MHz}$ ), observing the presence of 6 follicles in the right ovary $(\geq 0.5 \mathrm{~mm})$ Figure 1 . Ovarian stimulation is started on day 3 of the cycle using 300IU per day of menopausal gonadotropic hormone (Merapur $\AA$ ), from day three to eleven, and $0.25 \mathrm{mg}$ of cetrorelix per day (Cetrotide), from day eight to eleven, followed by the application of the hormone chorionic gonadotropin (Ovidrel) on day twelve of the cycle and on day 14 performing follicular aspiration, obtaining 2 oocytes in metaphase II: expanded and luteinized cluster cells and 1 oocyte in Metaphase I: less expanded cluster cells, using HTF HEPES Human Tubal Fluid (In Vitro. Care, Cat \# 2002-5, 500ml). The 3 oocytes were immediately vitrified (Kitazato Co, Tokyo, Japan).

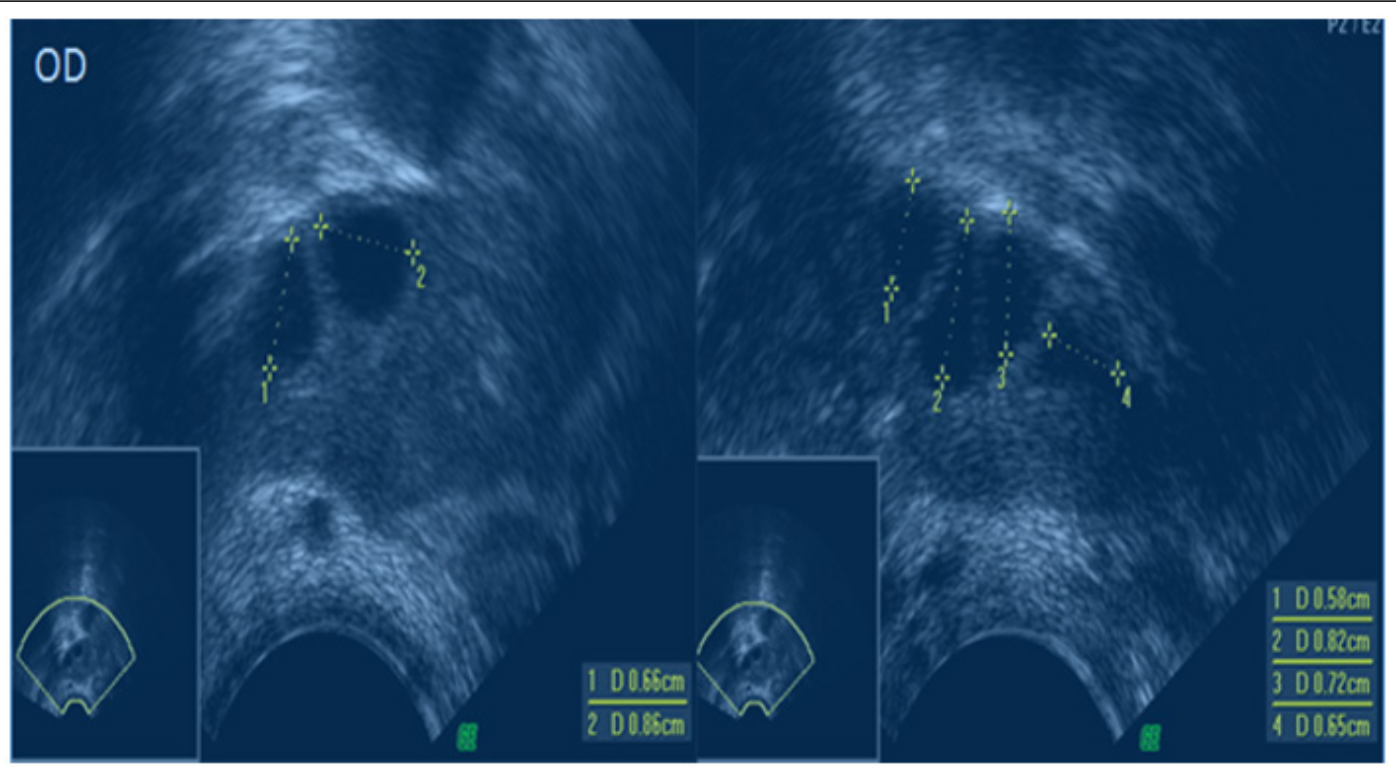

Figure 1: Shows $6 \mathrm{AFC}$ in the right ovary, larger than $5 \mathrm{~mm}$. Study performed with vaginal ultrasound (Philips, Affiniti 50, with a minimum of $7 \mathrm{MHz}$ ). 
Taking this first cycle into account, the patient is classified as a low idiopathic responder, including her in group 2 and subgroup 2a of the Poseidon criteria. The proposed treatment option for this type of patient is the application of higher doses of gonadotropins and longer stimulations. As ovarian stimulation started with high doses in the first cycle, due to the history of maternal age and her desire to vitrify oocytes, it was decided to change the medication from Merapur to Pergoveris while maintaining the same dose and proposed therapy with tissue stem cells Adipose autologous ADMSCs applied directly to the ovary as an adjuvant. The nature of the procedure to be performed is explained to the patient, and she accepts the written informed consent of the procurement of autologous AD-MSCs as an adjunct. Following, on day 14 of the menstrual cycle, the patient goes to the operating room, where the fat tissue is obtained, beginning with the selection of the obtaining surface, followed by antisepsis and application of local anesthesia. Obtaining 60cc of solid fat from which 40x106 of mesenchymal stem cells were obtained, which were diluted in platelet rich plasma, until reaching a volume in milliliters. Immediately after obtaining mesenchymal stem cells on day 14 of the cycle, the patient was placed in the lithotomy position, prior to the application of general anesthesia and with vaginal ultrasound (Philips, Affiniti 50, with at least $7 \mathrm{MHz}$ ) the ovaries, (Figure $2 \& 3$ ).

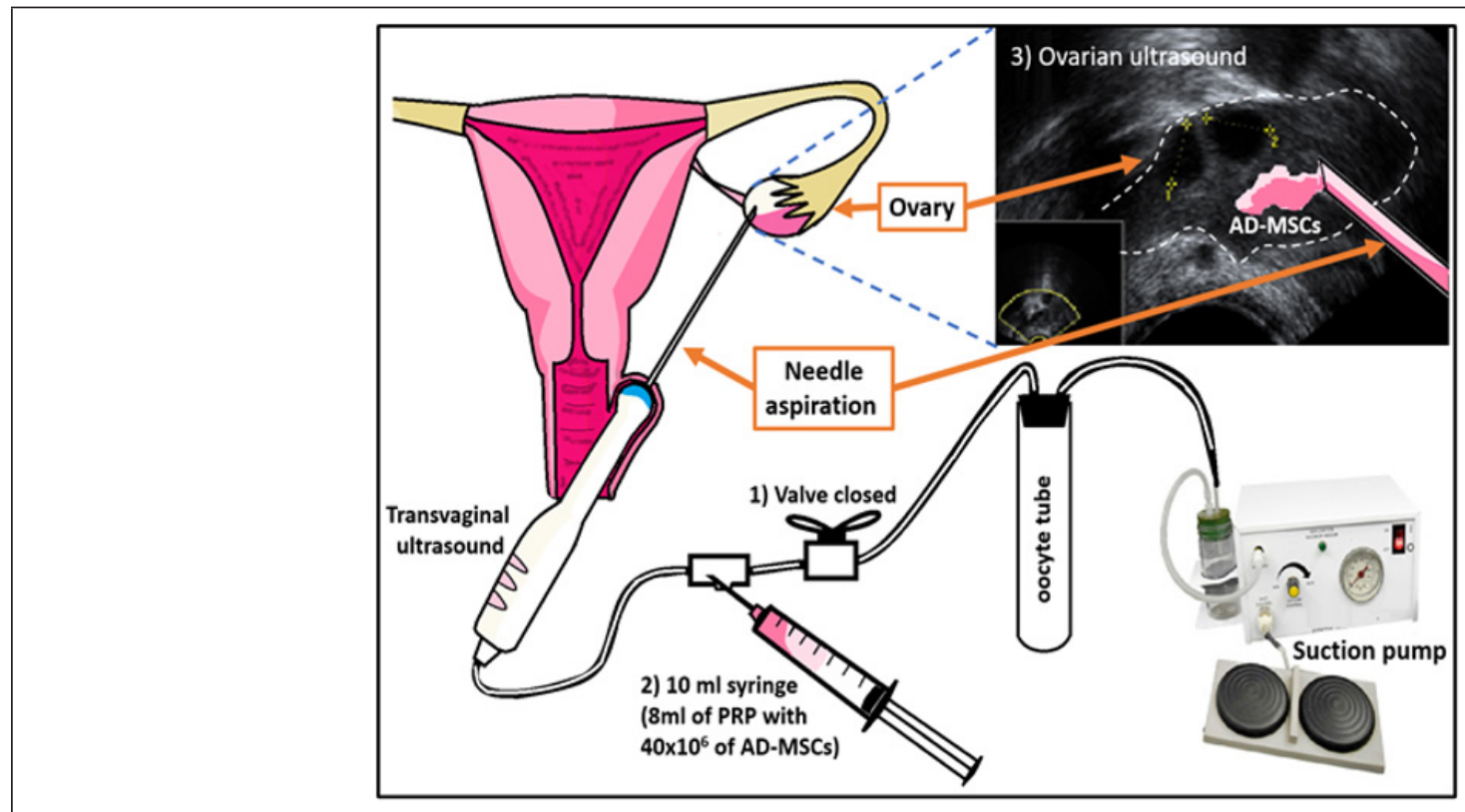

Figure 2: Technique; Application of PRP+AD-MSCs in the ovary

1. The aspiration system is closed to prevent PRP+AD-MSCs from going to the oocyte collection tuve 2. PRP + AD-MSCs are injected with the flow of the system directed to the ovary and

3. It is observed how PRP + AD -MSCs is deposited in the ovary.

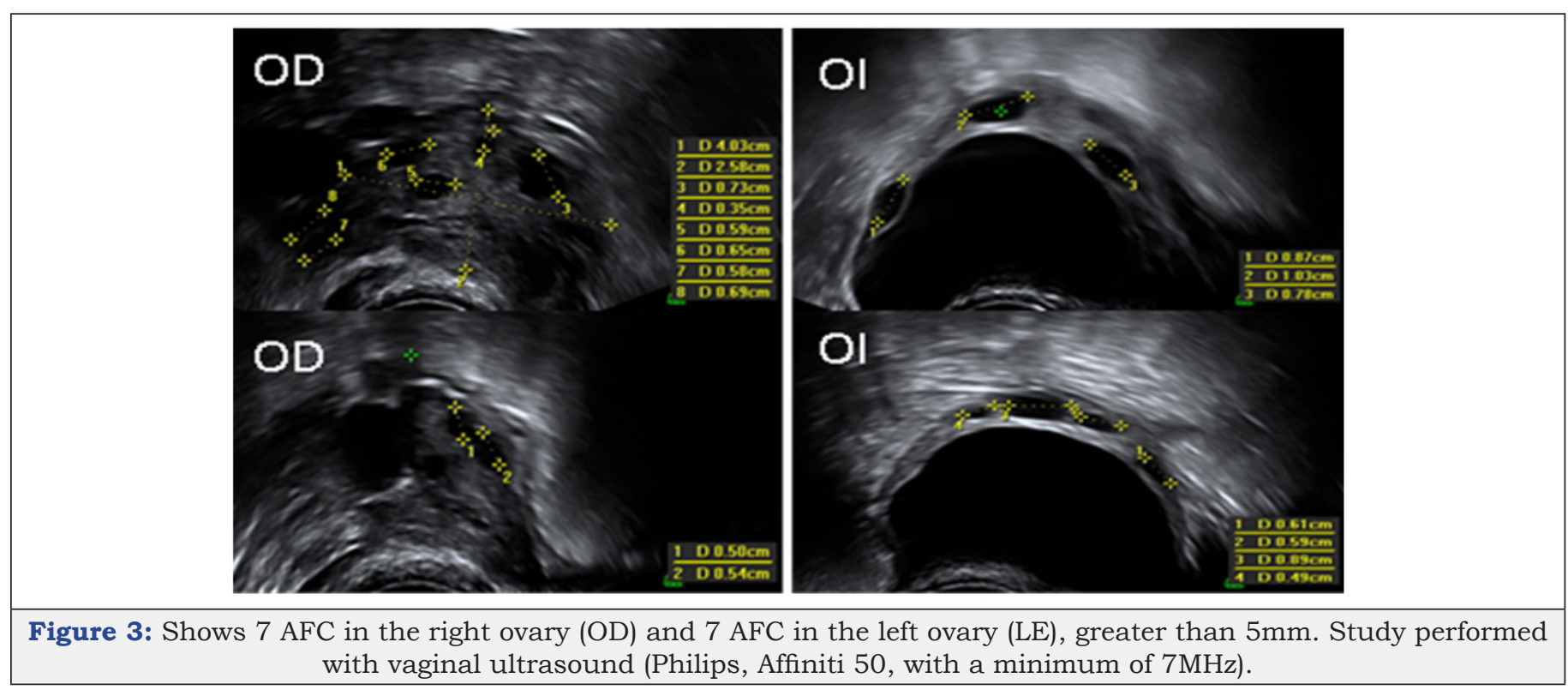


From this moment on, the patient makes the decision to devitrify the 3 oocytes from the first cycle (Kitazato Co, Tokyo, Japan) and, together with the 14 oocytes from the second cycle, fertilize them with donor semen (normozoospermia). Decision that was approved by the medical team because the number of oocytes obtained so far coincided with 2 , who reports that to obtain at least one euploid blastocyst, a minimum of 11 metaphase II oocytes is needed: cumulus cells expanded and luteinized. Of the 17 oocytes only 13 were fertilized, of which they belonged to the second cycle and only 3 developed to a blastocyst (morphology of the classification of the three blastocysts (1BE/BB, 1BH/BD and 1BH/ $\mathrm{CD}$ ). embryonic development G1 culture medium was used from fertilization of the oocyte until day 3 (Vitro Life, REF: 10128) and from day 3 to 5 they were cultured in G2 medium (Vitrolife, REF: 10132). The incubator used was a Mini-Incubator, Cook, Australia, using a fixed gas mixture with 5\% 02 and 7.5\% $\mathrm{CO}_{2}$. A biopsy was performed of the three blastocysts obtained, to carry out a preimplantation genetic study for aneuploidies by new generation sequencing. The result of the study reported one euploid embryo and two aneuploidies. For embryo transfer, the euploid embryo was taken and placed in the mother's uterus, resulting in a positive menopausal gonadotropin hormone beta subunit test of $6572 \mathrm{mUI} /$ $\mathrm{mL}$; A male newborn of $3120 \mathrm{~g}$ and height of $51 \mathrm{~cm}$ was obtained by caesarean section at 39.2 weeks of gestation with APGAR of 8/9, which was progressing adequately.

\section{Discussion}

FSH/FSHR signaling plays an important role in normal gonadal function by regulating follicular growth, estrogen production, and oocyte maturation; the alteration of FSHR function generates a complete block of follicular maturation $[8,12,17]$, it is observed in mice with deficit of follicle stimulating hormone receptors, who received intravenous therapy with stem cells obtained of bone marrow, an increase in the number of antral follicles (6 vs. 28), E2 "(10 vs. $40 \mathrm{pg} / \mathrm{mL})$ and higher expression of the follicle-stimulating hormone receptor compared to the control group; In our clinical case report, the improvement in the ovarian response was demonstrated, obtaining more follicles and achieving pregnancy, by improving the quality of the oocytes. For their part, 11, they report the case of a 45-year-old woman with perimenopause (infrequent menstruation), who underwent transfer of autologous stem cells extracted from the bone marrow, the presence of 2 oocytes in the right ovary and 2 oocytes in the left ovary after the eighth week of application, in addition to an increase in HAM fluid from 0.4 to $0.9 \mathrm{ng} / \mathrm{mL}$. 3 oocytes were captured, one of which was of good quality and which was transferred. On day 14, pregnancy was reported with chorionic beta hormone gonadotropin 1280 and at 6 weeks gestation with ultrasound.

The birth was given at 38 weeks of gestation and it was a girl 5 of $2.7 \mathrm{~kg}$; Autologous stem cells obtained from bone marrow were transplanted to 10 patients aged 26-33 years with premature ovarian failure and normal karyotypes, observing that $20 \%$ resumed menstruation 3 months after treatment and increased antimullerian hormone, obtaining only one of the pregnancy after 11 months of therapy. Resulting in the birth of a live $3.3 \mathrm{~kg}$ girl at 38 weeks gestation. The increase in oocytes and their quality in this case report, and in the other studies mentioned, may be due to the fact that mesenchymal stromal cells promote the recovery of ovarian function through the inhibition of apoptosis of cells of the granulosa and follicular atresia by increasing the expression of the follicle-stimulating hormone receptor in granulosa cells, allowing folliculogenesis to be carried out correctly 6-8. This is the first report of a live newborn in Mexico, where autologous ADMSCs were administered analogously to conventional treatment, for low idiopathic ovarian response in a patient included in group 2, subgroup 2a, of the Poseidon criteria.

\section{Conclusion}

In patients diagnosed with low ovarian response by Poseidon's criteria (Group 2, subgroup 2), the use of autologous AD-MSCs is an alternative to achieve a healthy pregnancy and newborn; Further studies are required for its greater acceptance.

\section{References}

1. Eftekhar M, Sadat E, Tabibnejad N (2018) Outcome of reproductive technology in different subgroups of poor ovarian responder fulfilling the poseidon criterian. Middle East Fertility Society Journal 23(4): 399403.

2. Esteves S, Yarali H, Ubaldi F, Carvalho JF, Bento FC, et al. (2020) Validation of ART calculator for predicting the number of metaphase II oocytes required for obtaining at least one euploid blastocyst for transfer in couples undergoing in vitro fertilization/intracyoplasmic sperm injection. Frontiers in Endocrinology 10: 917.

3. Ferraretti A, Marca A, Fauser B, Nargund G, Gianaroli L, et al. (2011) ESHRE consensus on the definition of poor response to ovarian stimulation for in vitro fertiization: The Bologna vriteria. Human Reproduction 26(7): 1616-1624.

4. Humaidan P, Alviggi C, Fischer R, Esteves S (2016) The novel POSEIDON stratification of low prognosis patient in assisted reproductive technology and its proposed marker of successful outcome. F1000Research 5(2911): 1-8.

5. Vaiarelli A, Cimadomo D, Ubaldi N, Rienzi L, Maria Ubaldi L, et al. (2018) What is new in the management of poor ovarian response in IVF? Current Opinion 30(3): 155-162

6. Buhler K, Conforti A, De Placido G, Esteves S, Fischer R, et al. (2016) A New more detailed stratification of low responder the ovarian stiulation: From a poor ovarian response to a low prognosis concept. Fertility and Sterility 105(6): 1452-1453.

7. Kamal S, Ramaraju G, Shashikant M (2020) Management strategies for POSEIDON Group 2. Frontiers in Endocrinology 27(11): 105.

8. Qin Y, Jiao X, Leigh J, Chen ZJ (2015) Genetic of primary ovarian insufficiency: New developments and opportunities. Human Reproduction Update 21(6): 787-808.

9. Edessy M, Hosni H, Shady Y, Waf Y, Bakr S, et al. (2016) Autologous stem cells therapy, the first baby of idiophatic premature ovarian failure. Acta Medica International 3(1): 19-23. 
10. Elfayomy A, Almasry S, El Tarhouny S, Magda A Eldomiaty (2016) Human umbilical cord blood-mesenchymal stem cells transplantation renovates the ovarian surface epithelium in a rat model of premature ovarian failure: Possible direct and indirect effects. Tissue and Cell 48(4): 1-13.

11. Ghadami M, El-Demerdash E, Zhang D (2012) Bone marrow transplantation restore follicular maturation and steroid hormones production in a mouse model for primary ovarian failure. Plos ONE 7(3): e32462.

12. Gupta S, Lodha P, Selva M, Tandulwadkar SR (2018) Role of autologous bone marrow-derived stem cell therapy for follicular recruitment in premature ovarian insufficiency: Review of literature and a case report of world's first baby with ovarian autologous stem cell therapy in a perimenopausal woman of age 45 year. Journal of Human Reproductive Sciences 11(2): 125-130.

13. Liu T, Li Q, Wang S, Chen C, Zheng J (2016) Transplantation of ovarian granulosa-like cells derived from human induced pluripotent stem cells for the treatment of murine premature ovarian failure. Molecular Medicine Reports 13(6): 5053-5058.

14. Yin N, Wang Y, Lu X, Liu R, Zhang L, et al. (2018) hPMSC Transplantation restoring ovarian function in premature ovarian failure mice is associated whit change of Th17/Tc17 and Th17/treg cell ratios trough the P13K/Akt signal Pathway. Stem Cell Res Ther 9(1): 37.

15. Yin N, Zhao W, Luo Q, Yuan W, Luan X, et al. (2018) Restoring ovarian function whit human placenta-derived mesenchymal stem cells in autoimmune-induced premature ovarian failure mice mediated by treg cells and associated cytokines. Reproductive Sciences 25(7): 1073-1082.
16. Andrzejewska A, Lukomska B, Janowski M (2019) Concise review: Mesenchymal stem cells: From roots to boost. Stem cells 37(7): 855-864.

17. Charles-de-Sá L, Ferreira AN, Maeda C, Borojevic R, Benati D, et al. (2015) Antiaging treatment of the facial skin by fat graft and adiposederived stem cell. Plastic and Reconstructive Surgery 135(4): 999-1009.

18. Elbuluk A, Einhorn T, Iori R (2017) A comprehensive review of stem cell therapy. JB and JS Reviews 5(8): e15.

19. Keun C, Jun L, Yoo S (2014) Therapeutic potential of human adiposederived stem cells in neurological disorders. Journal of Pharmacological Sciences 126(4): 293-301.

20. Macrin D, Joseph J, Arumugan A, Devi A (2017) Eminent sources of adult mesenchymal stem cells and their therapeutic imminence. Stem Cells review and Reports 13(6): 741-756.

21.Zakrzewski W, Dobrzynski M, Szymonovicz M, Rybak Z (2019) Stem cells: Past, present and future. Stem Cell Research \& Therapy 10(68): $1-22$.

22. Kang J, Yeon B, Cho S, Suh Y (2016) Stem cell therapy for Alzheimer's disease: A review of recent clinical trials. Journal of Alzheimers Disease 54(3): 879-889.

23. Leyva L, Ramentol C, Fernández T, Nicolau E (2017) Stem cells: A revolution in regenerative medicine. MEDISASN 21(5): 574-581.

24. Huhtaniemi I, Hovatta O, La Marca A, Livera G, Monniaux D, et al. (2018) Advances in the molecular pathophysiology, genetics, and treatment of primary ovarian insufficiency. Trends Endocrinol Metab 29(6): 400-419.

For possible submissions Click below: 